\title{
Commentary
}

\section{Opportunities for intervention and innovation in school food within UK schools}

\author{
JV Woodside ${ }^{1, *}$, A Adamson², S Spence ${ }^{2}$, T Baker $^{3}$ and MC McKinley ${ }^{1}$ on behalf of the \\ GENIUS (Generating Excellent Nutrition In UK Schools) network \\ 'Centre for Public Health, Queen's University Belfast, Institute of Clinical Science A, Grosvenor Road, Belfast BT 12 6BJ, \\ UK: ${ }^{2}$ Population Health Sciences Institute (Human Nutrition Research Centre), Faculty of Medical Sciences, Newcastle \\ University, Newcastle upon Tyne, UK: ${ }^{3}$ Charlton Manor Primary School, London, UK
}

Submitted 19 July 2020: Final revision received 2 November 2020: Accepted 11 November 2020: First published online 17 November 2020

\begin{abstract}
We have recently been funded by the UK Prevention Research Partnership (UKPRP) to develop a UK school food network. The overarching aim is to build a community working towards a more health-promoting food and nutrition system in UK schools (primary and secondary). Here we describe the current status of school food research, including a review of the literature supporting the healthpromoting schools approach and outline the opportunities for intervention and innovation establishment of the network present. Key potential school food research themes are described, and their prioritisation within the network, as well as network activities that have been planned, with the ultimate ambition of reducing socio-economic diet-related inequalities, and, consequently, noncommunicable disease risk.
\end{abstract}

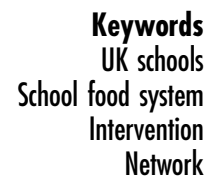

Diet is a key, modifiable health determinant. UK children's diets are high in saturated fat and sugar and low in fibre and fruit and vegetables. For example, only $8 \%$ of UK 11-18year-olds are meeting the '5-a-day' guideline, with socioeconomic differentials ${ }^{(1)}$. The quality of diet in childhood has been shown to impact on future development, educational achievement, health and well-being outcomes and also influences adult diet and non-communicable diseases risk (e.g. diabetes, CVD) in later life ${ }^{(2)}$. We need effective and sustainable ways of helping young people, particularly in areas of socio-economic disadvantage, access a better diet, as early modification in eating habits and behaviours will decrease risk of developing such conditions ${ }^{(3)}$.

Schools play a crucial role in improving children's health and are an obvious setting for population-level public health interventions ${ }^{(4)}$. They provide easy, and almost universal, access to pupils of various ages from across the social spectrum, with children spending approximately $40 \%$ of their time awake every week day at school. Food provided in schools could have a major influence on children's diet quality and has the potential to reduce inequalities in dietary intake ${ }^{(5,6)}$. Promoting the health and nutrition of the school food system can be seen as an essential activity that supports the goals of education. There are differences in how schools arrange their food provision and what they serve, between schools and UK regions, and this is not well understood. A systematic review of interventions to prevent childhood obesity highlighted, however, that establishing school environments and cultural practices that support children eating healthier foods throughout the day could be an effective intervention strategy ${ }^{(7)}$.

UK school food was deregulated in 1980, and, between 2000 and 2013, each nation re-introduced school food standards across primary and secondary schools. These standards varied in content and timetable for implementation, but are now compulsory (in all nations since 2013), except for English academies founded between 2010 and 2014. Evaluation and monitoring of standards, and the requirements for reporting of these, across the UK have varied in scope, detail and timing ${ }^{(8)}$. When standards have been

*Corresponding author: Email j.woodside@qub.ac.uk

(C) The Author(s), 2020. Published by Cambridge University Press on behalf of The Nutrition Society 
implemented, evaluations conducted in England suggest that the quality and nutritional value of food provided have improved, both within and beyond the school setting, and children from all socio-economic groups have benefitted $^{(9-11)}$.

There has, to date, been no formalised research, practice or policy network bringing UK school food stakeholders together to understand and share best practice. There is much to learn regarding the landscape and initiatives regionally that could be applied UK-wide. This required learning has been brought into sharp focus recently with the closure of schools during the COVID-19 pandemic, when the government response in terms of replacement support for those eligible for free school meals, and the heterogeneity with which alternatives were implemented, received much attention ${ }^{(12)}$.

It is critical to policy development that the value of investment, innovation and change in the school food system are measured and protected using planned, appropriate, robust and timely evaluations, working across government departments and between countries. Broader insight is needed to provide a foundation for future actions, in terms of activities ripe for innovation, intervention development/testing and effective routes to influence policy.

\section{School food system research: the health-promoting schools 'whole-school approach'}

The health-promoting schools (HPS) approach is advocated internationally to support and foster positive health behaviours $^{(13,14)}$.

Recognising that education and health are intrinsically linked, the WHO defines a HPS as 'a school that is constantly strengthening its capacity as a healthy setting for living, learning and working'. The WHO's HPS Framework ${ }^{(15)}$ advocates a 'whole-school approach' to promoting health via: provisions and activites relating to health-promoting school policies; the school's physical and social environment; the curriculum; family and community links and health services at the school ${ }^{(16)}$.

Recent reviews of the HPS approach have indicated not only some successes but also gaps in the evidence base ${ }^{(17-20)}$. Notably, activities to engage families and communities are weak, implementation in low-income settings is largely absent, most studies have focused on primary school-age children rather than adolescents and few interventions examine impacts on educational outcomes alongside health indicators. Furthermore, in terms of the robustness of the evaluation approach, evidence on sustainability, cost-effectiveness and contextual factors influencing effectiveness and implementation are lacking ${ }^{(17-20)}$.

Whilst sytematic review evidence has revealed some school-level factors that contribute to the successful operation of HPS (such as school leadership, support from parents, community support, culture and capactity $)^{(18,19)}$, information on the broader system-level elements that may impact local implementation barriers and support uptake of a HPS approach is limited ${ }^{(18)}$. Several barriers to developing sustainable and effective HPS are proposed ${ }^{(20)}$ including poor understanding of the complexity of schools and how they function as complex adaptive systems; lack of acknowledgement of the diversity between schools; inadequate shared information and experience about the HPS approach between schools and also between the education and health sectors and lack of effective interactions between schools and parents about health ${ }^{(20)}$.

An example of application of the HPS approach is the HEALTHY study ${ }^{(21)}$. It modified the total school food environment and observed positive changes in the nutritional quality of foods and beverages available in schools and modest improvements in fruit and water consumption ${ }^{(21)}$. Other components were physical education, behaviour change and social marketing. A systematic review of the impact of multi-strategy nutrition education programmes on the health and nutrition of adolescents ${ }^{(22)}$ also highlighted the important role of schools, stating that such interventions can significantly impact on adolescent nutrition, when the nutrition education is theoretically based and facilitated by school staff in conjunction with parents and families, and includes changes to the school food environment.

These whole school nutrition interventions exist that may be relevant to the UK setting; however, an understanding of context is essential to inform implementation and testing of approaches that have been developed in other countries. As highlighted by Turunen ${ }^{(16)}$, the factors that influence implementation of HPS interventions are numerous, variable and contextually influenced. It is essential to understand the complexity of the school food system in the UK in order to tailor and embed interventions appropriately in different contexts according to local needs ${ }^{(19)}$. There is also value in taking stock of current evidence and practice in order to develop a future research agenda that meets the needs of schools, pupils, parents and the wider community. Such an exercise has not been undertaken to date in the UK.

\section{Themes relevant to school food research}

A systems approach to school food, and considering schools as a complex adaptive system, may be pertinent to first understand their complexity and then allow the opportunities for innovation and intervention to be fully realised. However, even without such a methodology yet being applied, it is likely that the following themes will be relevant.

1. School food policies and standards: nutrient and foodbased standards across the UK need to be scoped, as does the evidence on which these are based. The policies and standards range according to age, are in place for school meals, but also for packed lunches 
and broader food provision in schools, e.g. vending machines (including beverages), breakfast clubs, after school clubs, tuck shops and snack foods. Provision of free school meals and the extent to which these are taken up, and actually consumed, also needs to be explored. Such a scoping exercise will identify gaps and explore heterogeneity in how standards are implemented and monitored. A similar, although higher level, analysis was conducted in 2014 across Europe, but did not capture the UK in detail ${ }^{(23)}$, whilst the previous UK-focused review is now outdated ${ }^{(8)}$. Opportunities to evaluate policy change need to be capitalised, e.g. introduction of universal infant free school meals and the role of advocacy considered.

2. School food procurement/provision: models of school food procurement/provision across the UK vary, with no consensus around what good practice is in terms of effectiveness, efficiency and sustainability. Metrics of good practice may include high uptake rates, increased diet quality, sustainability, reduced food miles, low levels of waste, both within the kitchen and plate waste in the canteen and cost efficiencies. Similarly, little is known about how savings, efficiencies and improvements in quality could impact in the long term on economic, educational and health outcomes. Increasing uptake of free school meals or improving meal quality with minimal financial impact on families could potentially reduce socio-economic inequalities.

3. The school food environment: long queuing times and concerns about hygiene and the quality of food offered are frequently mentioned barriers to school meal acceptability $^{(24)}$. Modifiable factors within the school food environment need to be explored, including timing of school meals, food sourcing practices and dining environment, i.e. a welcoming social environment and a lack of queues. How the school leadership team can intervene to successfully achieve such culture change must be determined, as should how catering staff training might help encourage children to eat higher nutritional quality foods. The feedback received from pupils, teachers and other staff members is likely to be of key importance to the success and development of the school food system, and therefore a direct and interactive feedback mechanism which supports concrete actions should be considered.

4. School food system data collection: limited data on school food are routinely collected throughout the UK, but a thorough understanding of what is available, for example, from local authorities in terms of school meal uptake, adherence to standards, would help to plan research with routine outcome assessment and allow potential linkage, for example, to educational, welfare and health outcomes. Harmonisation of data collection methods across the UK, including consideration of the key timepoints for data collection to allow the monitoring of trends over time and the impact of policy changes, would aid this. The use of technology to improve data collection, for example, exploiting big data from cashless meal card food purchases, should be considered.

5. Interventions in school settings: much work is already being conducted in UK schools ${ }^{(25,26)}$, but a thorough scoping review of current interventions to gain consensus on those likely to be successful would enable these to be tested on a larger scale. These could include not only whole school approaches but also age-specific meal size guidance for catering staff, interventions changing menu cycling and number/ type of options and utilising food choice architecture. Exploring how interventions could be implemented in practice, at scale and funding mechanisms will be important. Effects of such interventions on broader dietary change, for example, food intake at home, should also be examined. The theoretical basis (explicit or implicit) of existing or potential interventions needs to be explored, exploring the broad behaviour change routes that interventions may target, such as motivation, action, prompting or maintenance processes. Moreover, the specific 'mechanisms of action' within behaviour change processes will need to be identified (e.g. food literacy, confidence, knowledge and attitude for motivational processes, changing social norms and school food culture) and mapped onto the existing and potential tools and strategies for behaviour change to arrive at an adaptable theory-based toolkit with potential for cross-translation and testing in different school-based contexts.

\section{The GENIUS school food network}

The UKPRP-funded GENIUS network ${ }^{(27,28)}$ will consider the food system across preschool, primary and secondary settings and include all school food provision, within canteens, vending machines, shops and home-prepared packed lunches, and also the school fringe environment. The overarching aim of this network is to build a community to work towards a more health-promoting food and nutrition system in UK schools. Specific objectives include the development of a network of academics and non-academics across the UK actively researching and influencing the school food system, the use of a range of methodologies, including systems mapping and network analysis, to understand the current UK school food landscape, including similarities and differences, areas of best 
practice, and, finally, the exploration of opportunities for population- and system-level interventions that will positively impact diet quality and inequalities.

The network brings together an interdisciplinary team, including nutrition, epidemiology, public health, systems science, sensory science, health economics, health informatics, health psychology, education, planning and policy, to comprehensively examine school food in its broadest and most complex sense. Inclusion of non-academic project partners from across the UK will prioritise stakeholder/user engagement and allow the co-development of research priorities. The work of the network is at an early stage but, given the likely themes listed above, and early discussion amongst network members, Figure 1 demonstrates the themes with some consideration of how they inter-relate and how they might be prioritised.

\section{Conclusion}

The GENIUS network will bring together a wide range of UK school food stakeholders to build a better understanding of the school food system, innovate, co-create and share best practice. Understanding the current food system and building network expertise will advance research to better inform policy and good practice around food in

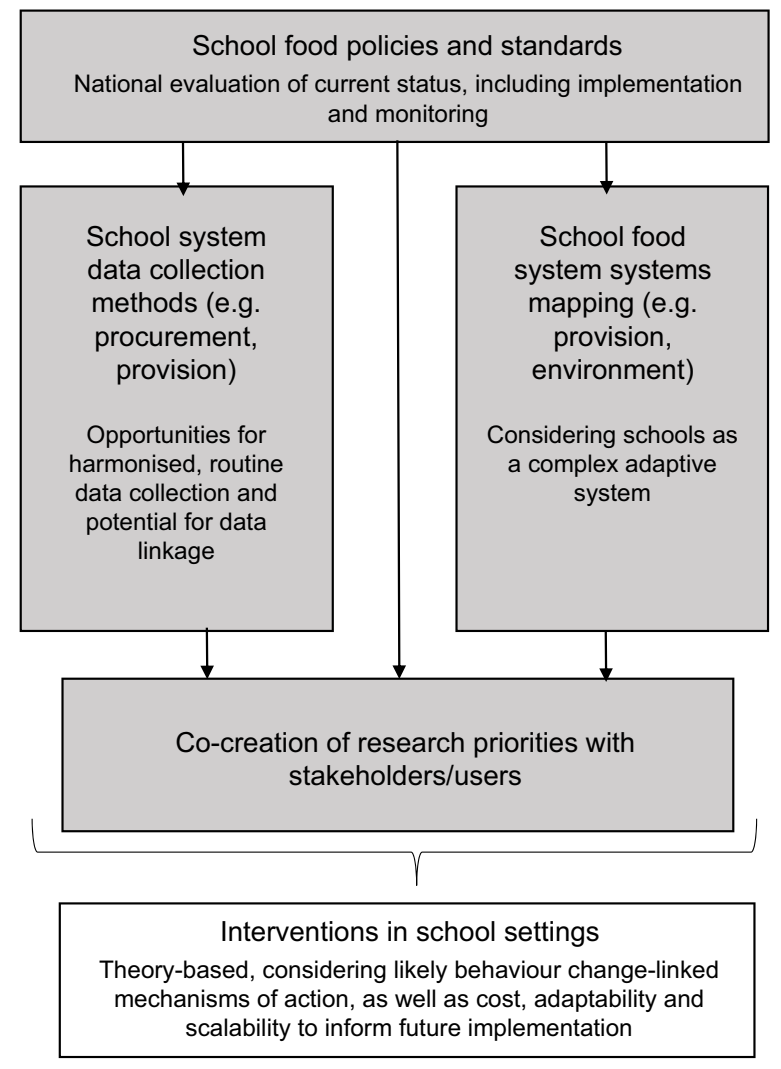

Fig. 1 Themes relevant to school food research. $\square$, Prioritised research themes and activities within the GENIUS network schools. The co-production of priorities for research and changes in practice will ensure that the activities of the network are relevant for beneficiaries (families with schoolage children), stakeholders (teachers, principals, catering staff and policymakers) and are, thus, more likely to result in meaningful and sustained changes in policy and practice. The network ultimately aims to effect an improvement in school food quality, normalise evidence-based healthy food practices within school settings and change school food culture and practice, creating healthier school food environments which are accessible to all, reducing socioeconomic diet-related inequalities, and, consequently, non-communicable diseases risk.

The GENIUS network consists of:

Jayne Woodside, Michelle McKinley, Ciaran O'Neill, Laura Dunne, Ruth Hunter, Queen's University Belfast; Ashley Adamson, Suzanne Spence, Newcastle University; Laura Johnson, Angeliki Papadaki, University of Bristol; Stephanie Chambers, University of Glasgow; Charlotte Evans, Hannah Ensaff, University of Leeds; Julie Lovegrove, Lisa Methven, Jeremy Spencer, Kate Harvey, University of Reading; Annie Anderson, University of Dundee; Sinead Brophy, Swansea University; Kevin Morgan, Cardiff University; Stephan Dombrowski, University of New Brunswick.

\section{Acknowledgements}

Acknowledgements. The authors wish to acknowledge the administrative support provided by Ms Angela Mullan. Financial support. This work was supported by the Medical Research Council (grant number MR/S03756X/ 1). Conflict of interest. The authors declare no conflicts of interest. Authorship. J.V.W. is PI of the GENIUS network and produced the first draft of the commentary. A.A., S.S., T.B. and M.McK. provided feedback and revised this first draft. All authors approved the final version of the manuscript. Ethics of buman subject participation. This commentary does not describe research involving human participants.

\section{References}

1. https://www.gov.uk/government/collections/national-dietand-nutrition-survey (accessed July 2020).

2. Craigie AM, Lake AA, Kelly SA et al. (2011) Tracking of obesity-related behaviours from childhood to adulthood: a systematic review. Maturitas 70, 266-284.

3. Bennett BJ, Hall KD, Hu FB et al. (2015) Nutrition and the science of disease prevention: a systems approach to support metabolic health. Ann NY Acad Sci 1352, 1-12.

4. Marmot M (2010) Fair society, healthy lives: the Marmot review: strategic review of health inequalities in England post-2010. Department for International Affairs, UK Government. ISBN 9780956487001. 
5. Moore GF, Murphy S, Chaplin K et al. (2014) Impacts of the primary school free breakfast initiative on socio-economic inequalities in breakfast consumption among 9-11-year-old schoolchildren in Wales. Public Health Nutr 17, 1280-1289.

6. Spence S, Matthews JN, White M et al. (2014) A repeat crosssectional study examining the equitable impact of nutritional standards for school lunches in England in 2008 on the diets of 4-7y olds across the socio-economic spectrum. Int J Behav Nutr Phys Act 11, 128.

7. Bleich SN, Vercammen KA, Zatz LY et al. (2018) Interventions to prevent global childhood overweight and obesity: a systematic review. Lancet Diabetes Endocrinol 6, 332-346.

8. Adamson A, Spence S, Reed L et al. (2013) School food standards in the United Kingdom: implementation and evaluation. Public Health Nutr 16, 968-981.

9. Spence S, Delve J, Stamp E et al. (2014) Did school food and nutrient-based standards in England impact on 11-12Y olds nutrient intake at lunchtime and in total diet? Repeat crosssectional study. PLoS One 9, e112648.

10. Spence S, Delve J, Stamp E et al. (2013) The impact of food and nutrient-based standards on primary school children's lunch and total dietary intake: a natural experimental evaluation of government policy in England. PLoS One 8, e78298.

11. Evans CEL, Melia KE, Rippin HL et al. (2020) A repeated cross-sectional survey assessing changes in diet and nutrient quality of English primary school children's packed lunches between 2006 and 2016. BMJ Open 10, e029688.

12. https://policystudies.blogs.bristol.ac.uk/2020/06/01/lockdownlunches/ (accessed October 2020).

13. Langford R, Bonell C, Jones H et al. (2015) The World Health Organisation's Health Promoting Schools framework: a Cochrane Systematic review and meta-analysis. BMC Public Health 15, 130.

14. Wang D \& Stewart D (2012) The implementation and effectiveness of school-based nutrition promotion programmes using a health-promoting schools approach: a systematic review. Public Health Nutr 16, 1082-1100.

15. World Health Organisation (1997) Promoting Health Through Schools. Report of a WHO Expert Committee on Comprehensive School Health Education and Promotion. WHO Technical Report Series 890. Geneva, Switzerland.

16. Turunen H, Sormunen M, Jourdan D et al. (2017) Health promoting schools - a complex approach and a major means to health improvement. Development of health promoting schools in the European region. Health Promotion Int 32, 177-184.

17. Langford R, Bonell C, Komro K et al. (2017) The health promoting schools framework: Known unknowns and an agenda for future research. Health Educ Behav 44, 463-475.

18. McIsaac JL, Hernandez KJ, Kirk SF et al. (2016) Interventions to support system-level implementation of health promoting schools: a scoping review. Int J Environ Res Public Health 13, 200.

19. Hung TTM, Chiang VC, Dawson A et al. (2014) Understanding of factors that enable health promoters in implementing health-promoting schools. PLoS One 9, e108284.

20. Keshavarz N, Nutbeam D, Rowling L et al. (2010) Schools as social complex adaptive systems: a new way to understand the challenges of introducing the health promoting schools concept. Soc Sci Med 70, 1467-1474.

21. Gillis B, Mobley C, Stadler DD et al. (2009) Rationale, design and methods of the HEALTHY study nutrition intervention component. Int J Obesity 33, S29-S36.

22. Meiklejohn S, Ryan L \& Palermo C (2016) A systematic review of the impact of multi-strategy nutrition education programs on health and nutrition of adolescents. JNutrEduc Behav $\mathbf{4 8}$, 631-646.

23. http://publications.jrc.ec.europa.eu/repository/bitstream/ JRC90452/lbna26651enn.pdf (accessed July 2020).

24. Payán DD, Sloane DC, Illum J et al. (2017) Perceived barriers and facilitators to healthy eating and school lunch meals among adolescents: a qualitative study. Am J Health Behav 41, 661-669.

25. Nathan N, Janssen L, Sutherland R et al. (2019) The effectiveness of lunchbox interventions on improving the foods and beverages packed and consumed by children at centrebased care or school: a systematic review and meta-analysis. Int J Behav Nutr Phys Act 16, 38.

26. Langford R, Jago R, White J et al. (2019) A physical activity, nutrition and oral health intervention in nursery settings: process evaluation of the NAP SACC UK feasibility cluster RCT. BMC Public Health 19, 865.

27. https://ukprp.org/what-we-fund/genius/ (accessed July 2020).

28. https://geniusschoolfoodnetwork.com/ (accessed July 2020). 